\title{
Computational Modelling of Image Coding using ROI based Medical Image Compression
}

\author{
Suma \\ Research Scholar, \\ Dept. of Elect and Comm. Engg. \\ Vidya Vikas Institute of Engineering and \\ Technology, Mysore, India
}

\author{
V Sridhar, Ph.D. \\ PES College of Engineering \\ Mandya, India
}

\begin{abstract}
In the age of digital medical imaging communication and robotic transmission of real-time image for robot guided operations in constrained bandwidth is a challenging task. The issue of compression, in medical images, is the prime focus of this study. The study has aimed to perform an investigation on the frequently adopted region-of-interest scheme called as MAXSHIFT. The design principle of this standard encoding technique allows encoding and highly prioritizing only the region of interest and then emphasis on the background (nonregion of interest area). The system allows the deployment of multiple and randomly shaped region of interest within the medical images using randomized weights for emphasizing each element of ROI. Supported by the discussion on some of the prior research work, and how this study is motivated, the present manuscript illustrates the experimental phases of implementing MAXSHIFT on two dimensional medical images. In order to check the robustness of the algorithm, the performance parameters such as bit per pixel (BPP) and Signal to Noise Ratio (SNR) is being evaluated on enormous medical images.
\end{abstract}

\section{Keywords}

Medical Images, Compression, JPEG2000, MAXSHIFT, Region of Interest,

\section{INTRODUCTION}

The field of medical image processing is on the constant attention for the researcher especially from the last decade. Basically, medical images pertaining to the radiological images just like computed tomography or the Magnetic resonance imaging exhibits the clinical images of the human body in digital format. Since these imaging techniques generate substantial quantity of data, compression is, therefore, critically required for storage and communication purposes. The compression technique furnishes a very higher degree of compression rate and is also associated with possible loss of significant information which is valuable from medical diagnosis viewpoint. However, when such medical images are captured from the diagnosis viewpoint, not all the areas captured are of clinical importance. In the complete frame of medical image, only a significant portion of the image may be of significant interest. It was seen that a usual medical image (say x-ray) consists of $2048 \times 2560$ pixels in dimension [1], which consumes a higher degree of memory in the storage to save it and then analyse it. This massive information of medical image posses a great challenge when it comes to storage and transmission in the area of telemedicine. Although disk storage is not a bigger issues in current era due to availability of efficient storage mechanism. But, the problem arises when everyday thousands of such images are stored in large scale chain of pathological reports reaching the saturation level of the disk storage. With the prevalence of telemedicine in majority of large chains of hospitals, image transmission has become one of the challenges when it comes to transmitting a massive size of image on limited bandwidth. This causes delay as well as loss of significant clinical information of the medical image. Hence, owing to the voluminous size of such medical image, compression technique is inevitable in the area of medical image processing.

The compression techniques applied in medical image processing usually consists of three significant components e.g. i) decorrelation algorithm, ii) primary compression engine, and iii) formatting environment. The decorrelation algorithm uses the data redundancies in medical image using multi-resolution frameworks, while primary compression engine performs minimization of medical image size. Formatting environment ensures the compatibility of the image file in the receiver machine. On the other hand, multi-resolution models decorrelate image data by using a transform, such as discrete wavelet transforms (DWT) or discrete cosine transforms (DCT). In the proposed study, we highlight a standard medical compression technique using the most frequent standard of MAXSHIFT method. The primary contribution of the proposed system is as follows: i) to consider medical image database and perform region of interest to truncate the possibility of image evaluation of less-clinical importance, ii) to apply discrete wavelet transform for decomposing the selected Region of interest for further supportability in compression technique, iii) to apply entropy coding on the accomplished coefficients, iv) to perform MAXSHIFT method by scaling up the selected region of interest for extracting the highest possible information, and v) to perform evaluation of the resultant image using SNR and BPP. Section 2 gives an overview of related work that identifies all the major research work being done in this area. The problem identification is discussed in Section 3. Section 4 highlights the proposed system followed by implementation \& result discussion on Section 5. Section 6 makes some concluding remarks about the paper.

\section{RELATED WORK}

This section presents some of the prior research work that has been introduced for the purpose of mitigating issues of medical image compression. A hybrid model of lossless compression of the selected region of interest was studied by Gorturk et al. [2] considering maximized data rate along with efficient motion compensation and lossy compression on the non-selected region. The experimentation were carried out using medical images and the outcome of the study shows that it is better than other techniques of compression e.g. vector quantization and principal component analysis.

Similar study on medical images using region of interest was done by Loganathan et al. [3], who have used image segmentation process exclusively on lossless compression on cropped portion of the medical images. The author has also 
evaluated for lossy compression for non-cropped portion of a medical image. The author claimed that such techniques can have higher technical adoption in the medical image processing.

A novel concept of tree-based scheme was proposed by Babu et al. [4]. The author has termed the scheme as embedded zero tree wavelet algorithm which is completely motivated from progressive medical image compression technique. Study on similar direction was carried out by Bairagi et al. [5], where the author has considered DICOM image and integer wavelet transform. The result was found with minimized distortion after applying compression technique on the non-cropped region of the complete medical images. The study has successfully achieved its goal to truncate the noisy background and rebuild the medical image losslessly.

Some of the studies are found to consider JPEG2000 standards of medical image for performing compression. Evidence of such implication can be seen in the study performed by Sridevi et al. [6]. The author used coding on region of interest using JPEG2000 scaling method. The author has also used shape based approach for image coding using discrete cosine transform and sub band block of hierarchical partitioning.

Use of digital watermarking technique for the purpose of preventing rate distortion on the particular image data exists in region of interest was seen in the study of Wakatan [7]. The model used in the study successfully embed signature information with each other in all the non-cropped area making it easier and secure to understand the region of interest coding.

Use of wavelet based technique was found in the algorithm presented by Kumar et al. [8]. The author has used Contourlet based medical image compression technique. The author highlighted that superlative performance of image compression can be seen on Contourlet based transform technique when integrated with bidirectional filter banks and Laplacian pyramid. The author has segregated the region of interest from the background of the entire image using fuzzy $\mathrm{C}$ means algorithm which is followed by Contourlet based transformation. The outcome shows image with better visual quality.

Artificial compression framework was also surfaced in the research archival. One of such significant study was done by Thomas et al. [9]. The author has used this technique to perform faster transformation of a medical image exclusively for telemedicine. The study was also found to use fast and efficient discrete curvelet transforms with adaptive entropy coding particularly for lossless compression. The outcome of the study was found to have minimized error rate and optimal compression.

A simple algorithm to compress medical image was presented by Chavad et al. [10] using US medical image database. Advance application of medical images (MRI) was studied by Naidu et al. [11] for performing lossless image compression. The author has used three dimensional MRI dataset for performing coding and decoding of selected region of interest. The outcome of the study was verified by scalable SNR value. A combination of segmentation and lossless compression technique for medical image was presented by Arun and Murugan [12]. The technique uses wavelet based transform for performing compression. Similar line of study was also conducted by Kaur et al. [13]. The author has used the same principles in optimal manner for accomplishing their results of significant compression.
A context based technique was presented by Sridevi et al. [14] as an alternative of compression technique for medical images. The author has used Set partitioning in Hierarchical trees or commonly known as SPIHT algorithm using context based features for medical images. Using region of interest schema, the author has now extended it to call it as contextual region of interest that redefines the compression technique to be performed on conventional region of interest schemes. The outcome of the study shows higher image resolution and efficient compression technique.

Various studies exists in the literatures that has combinely uses the lossless and lossy compression technique for accomplishing higher rate of compression ratio and efficient perceptual quality in the cropped region [15]. In lossy methods, some information is lost as the high compression ratio is the main target. In lossless methods, the exact original image is reconstructed from the compressed image. Mateika et al., [16] analyzed medical image compression in popular compression formats. For the compression format comparison, there is proposed image quality evaluation algorithm based on the calculation of the mean exponent error value. The image quality evaluation experiment analyzed the distribution of the errors in medical images and explained the causes determining better than usual compression effect. Hence, majority of the standard study [17][18] have introduced ROI mask for identifying which coefficient have to be transmitted exactly in the order for the receiver for the purpose of reconstructing the ROI. Differing from the review of literatures, it has been seen that various researcher highly adopts MAXSHIFT method. Penedo et al. [19] in their study titled 'Region-Based Wavelet Coding Methods for Digital Mammography' has discussed the significance of usage of MAXSHIFT. The author commented that object shape information will be required in MAXSHIFT method to transmit first into the network channel. Silva [20] has conducted a study 'Advances in medical image compression: Novel schemes for highly efficient storage, transmission and on demand scalable access for 3D and 4D medical imaging data' where the author has significantly used MAXSHIFT method to carry out the compression of medical images. The significant findings of the study made some significant suggestion which acts as guidelines to the proposed study. The entropy decoder in 3D JPEG2000 standards should have potential to decode a large quantity of bit planes to scale up by MAXSHIFT. The author has added that existing decoder implementation just confirms that JPEG2000 standards are not efficient enough to perform decoding of massive volume of bit planes. Hence, this phenomenon also suggests that MAXSHIFT method is definitely not an appropriate procedure for accomplishing lossless reconstruction of the radiological data with volume of interest decoding capabilities. Similar discussion about the usage of MAXSHIFT has been done by Anastassopoulos [21], Chen et al. [22], and most recently by Pawadshetty [23]. Hence, it can be seen from the literatures that adoption of MAXSHIFT for performing medical image compression by ROI approach is quite higher. Hence, the proposed study chooses to implement MAXSHIFT method for the similar cause, but will emphasize on the image resolution as well as significant information by means of PSNR and BPP. Hence, it can be seen that there are some significant studies that has attempted to perform the medical image compression using various techniques. The previous paper A Review of the Effective Techniques of Compression in Medical Image Processing summarizes all these issues in details [26]. 


\section{PROBLEM IDENTIFICATION}

In analysis of related work studies in the previous sections, it has been concluded that the challenge for digital medical image compression is that even though high image compression rate is desired, usability of reconstructed images is dependent on important characteristics of the original image that requires preservation for correct diagnosis [3]. Medical image compression techniques are classified into lossy and lossless. The lossy approach accomplishes maximum compression ratios through different tactics efficiently retaining visually significant information. The lossless technique gets the unassuming compression ratios due to conservative maintenance of information. However, it was seen that, in medical and scientific imaging, the lossless compression technique is usually deployed due to the possible issue of losing valuable and sensitive information. It is considered that loss of minor details leading to subsequent medical diagnosis/scientific post-processing can result in legal liabilities/incorrect diagnoses. However, the medical image compression community argues that lossy medical image compression is necessary and helpful in the long run. They contend that technology must be considered to enhance lossy technique as the major chunk of medical data requires remote storage of hard-copy films, frequently resulting in loss or damage and always needing time to locate and transfer. Thus, instead of thinking in terms of lossless-lossy dichotomy, alternative ways must be sought to compromise conflicting requirements. In such a scenario more users, in medical and scientific communities have started to accept "nearlossless" methods as a tradeoff between compression ratios and distortion to enable achievement of higher compression ratios with limited distortion to ensure the accuracy for specific purposes. Medical imaging impacts medicine especially diagnosis and surgical planning. Imaging devices generate a large amount of data for each patient requiring storage and efficient transmission. Present compression schemes have high compression rates when quality loss is afforded. However, the physicians cannot afford deficiencies in image regions which are important, known as regions of interest (ROIs). An approach that brings high compression rates accompanied by good quality in ROI is highly required. The common idea is to preserve the quality in diagnostically critical regions while allowing lossy encoding of other regions. The proposed study targets to test the Region of Interest Scheme of coding for ensuring the deployment of multiple and randomly shaped ROIs in images, with random weights illustrating the importance for each ROI including background so that latter regions can be represented by varying levels of quality. Hence, the proposed system attempts to extract the information of the signal quality of the medical image by applying standard techniques. The prime purpose of the study is to check the efficiency of the standard techniques of performing medical image compression using region of interest scheme.

\section{PROPOSED SYSTEM}

The proposed system focuses on the significance of the Region of Interest scheme for performing medical image compression. The schematic architecture of the proposed study is highlighted below:

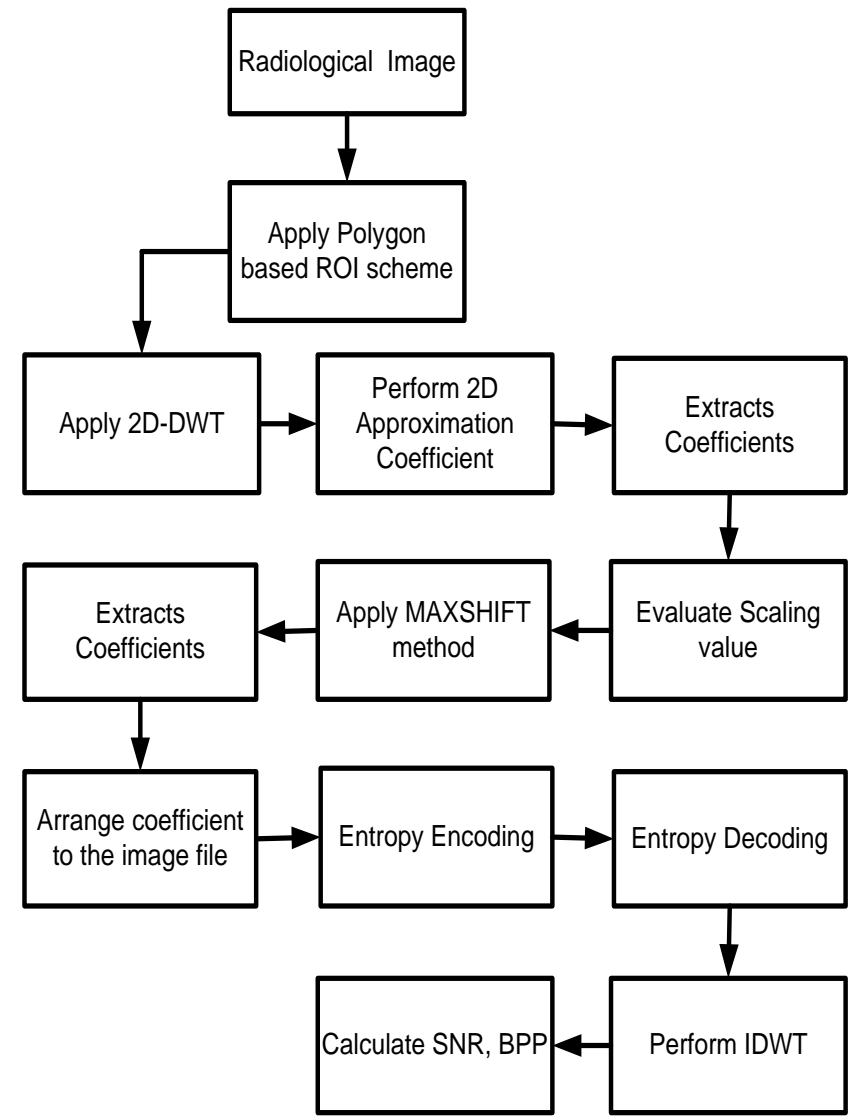

Fig 1: Schematic Architecture of the proposed study

Table 1. Selection of ROI from medical images

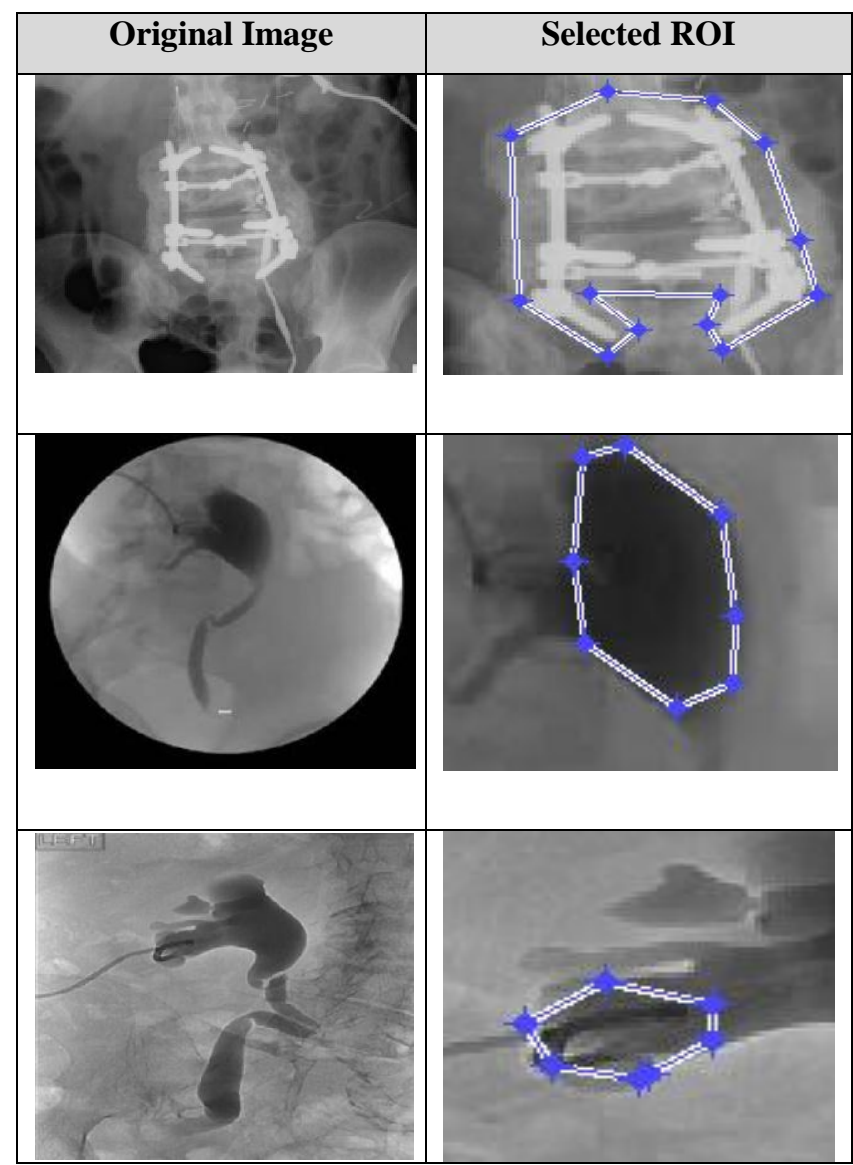



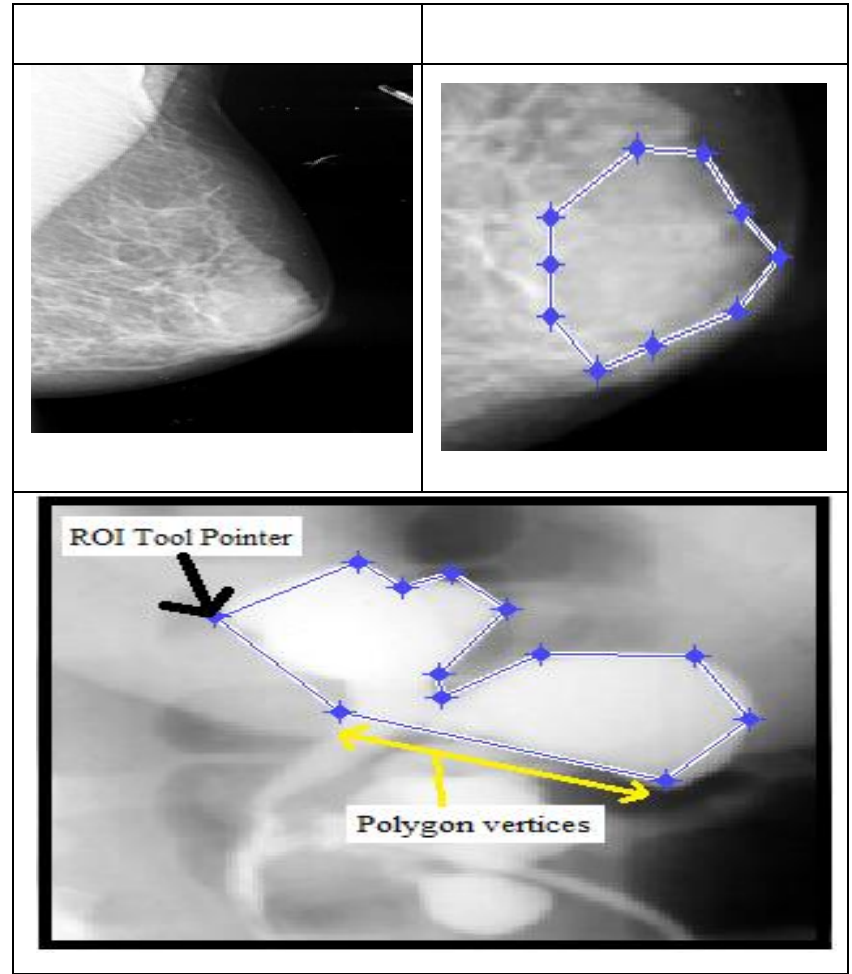

The study chooses to select ROI scheme as it is obvious that the physicians always need to emphasize the study on the specific portion of the radiological images (MRI image, PET scan images, CT scan image, etc.) in spite of studying the entire radiological images. Hence, there are two regions (I) ROI and (II) background region. The problematic factor of the phenomenon is that such ROI portion will be required to be encoded at the superior visual standards compared to the background region of an image. The prime reason behind this statement is that while choosing to discuss any applications related to medical image processing e.g. storage, encryption, or transmission, these ROI portions are given higher degree of priority to transmittance while background regions are always given lower degree of importance. Referring to the previous section, various schemes like DCT, DWT, etc. basically transformation based schemes, which when applied always gives the higher level of priority to the ROI portion as compared to the background. Therefore, there is an effective need of mathematically based approach which can identify the coefficient needed for the rebuilding process of ROI portion. Fig.1 exhibits the different essential functionality of the proposed system where the major stress is on performing compression technique on medical images using region of coding. The input of the system is any medical images usually in grayscale format, which is binarized and then it is subjected to user selection using ROI. The system deploys a function that is used to mark a region of interest in a polygonal shape as exhibited in Table 1. The function for ROI returns a binary image that can be used as a mask for mask filtering. Mask is a matrix designed for storing the convolved values of an input image. Once the region of interest is selected, the area is subjected to discrete wavelet transformation. The selected portion of the region of interest is evaluated on multiple levels of discrete wavelet transform that uses a series of filters. The initial part of the selected ROI image is subjected to low pass filters with impulse response $g$ that results in convolution of two signals $\left(1^{\text {st }}\right.$ signal $(x) \rightarrow$ selected ROI and $2^{\text {nd }} \operatorname{signal}(\mathrm{g}) \rightarrow$ impulse response). Therefore, convolution performed in intermediately steps of discrete wavelet transform may be represented as,

$$
y[n]=(x * g)[n]=\sum_{k=-\infty}^{\infty} x[k] g[n-k]
$$

After performing convolution as shown in eq.(1), the selected ROI is further subjected to decomposition using high-pass filters $h$. Therefore, the resultant furnishes the elaborated information about the coefficients from high-pass filters as well as the approximation coefficients from low-pass filters. The system also ensures that both the filters should be highly correlated with each other. By using the above mentioned step, only a partial section of the frequencies of the selected ROI has been removed, whereas remaining partial section of the image could be further removed using Nyquist's rule. The filtered resultant is further sub-sampled by 2 as shown below,

$$
\begin{aligned}
& y_{\text {low }}[n]=\sum_{k=-\infty}^{\infty} x[k] h[2 n-k] \\
& y_{\text {high }}[n]=\sum_{k=-\infty}^{\infty} x[k] g[2 n-k]
\end{aligned}
$$

The above representation of signal decomposition has halved the time resolution as only partial portion of each filter resultant represents the actual selected ROI image. Interestingly, as every resultant has half the band of frequency of the input image, so the resolution of the signal (image) frequency has been now doubly maximized.

Therefore, it has been seen that the applying wavelet transform is usually done at the encoding module, and the outcome of the coefficient are usually not related to the region of the interest that are scaled down. The estimation of the ROI mask is done in the following fashion - Consider $\mathrm{X}^{\mathrm{n}}$ as the wavelet domain of considered medical image and $\delta \in \mathrm{X}^{\mathrm{n}}$ as the selected region of interest. Therefore, the characteristic function $\psi \delta(\mathrm{x})$ can be designated as:

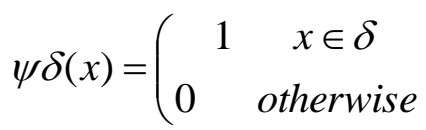

Then the mask of region of interest will be produced according to:

$$
\mathrm{g}_{\mathrm{i}}(\mathrm{x})=\left(\mathrm{W}_{\mathrm{i}} \cdot \mathrm{x} \delta\right)(\mathrm{x})+\mathrm{I}_{\mathrm{i}} \mathrm{x} \delta(\mathrm{x}), \mathrm{i} \in \mathrm{A}
$$

\begin{tabular}{|c|c|c|}
\hline Background & Region of Interest & Background \\
\hline MSB & 8 & MSB \\
\hline & 7 & \\
\hline & 6 & \\
\hline & 5 & \\
\hline & 4 & \\
\hline & 3 & \\
\hline & 2 & \\
\hline LSB & 1 & LSB \\
\hline
\end{tabular}

where $W_{i}$ will designate the wavelet operator for the $i^{\text {th }}$ subband, while $A$ is the index set of all sub-bands, and $I_{i}$ is the identity operator equipped with the down-sampling operation respectively [25].

Fig 2: Overall Image Compression 


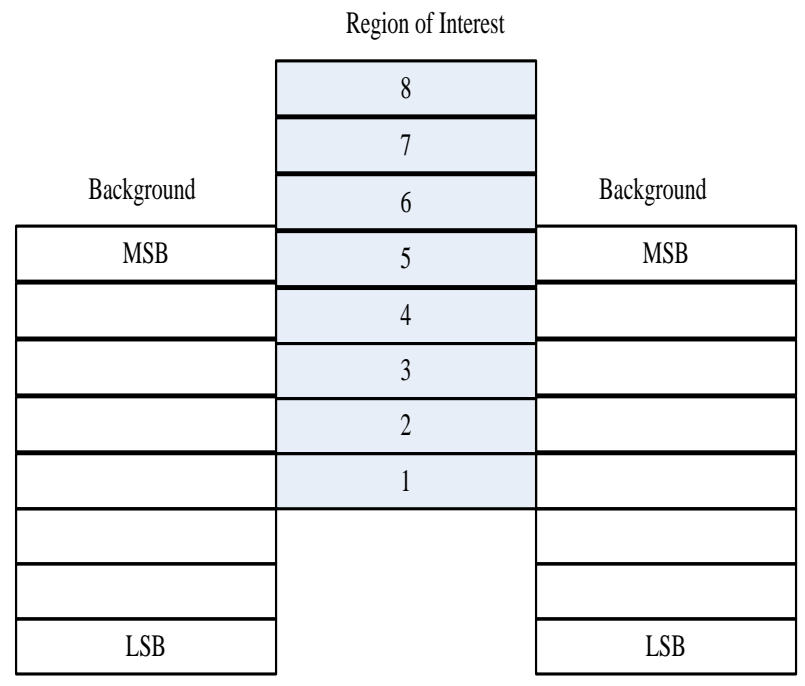

Fig 3: ROI scaling operations

The proposed system initially uses a compression method which is represented pictorially as in Figure 2. Using region of interest scheme, the proposed system scales up the region of interest where ROI portion of the image is coded in code stream without considering the remaining portion of the image as shown in Fig.3. We then apply MAXSHIFT method for producing the mask for region of interest portion of the medical image. The system then finds the scaling value that is higher than or equal to the maximum value of bit planes for any background coefficient in any code block in the existing components. Finally it scales down all background coefficient furnished by the mask in preliminary step by deploying scaling values. Finally, the code-stream is incorporated with scaling values. The entire operation of MAXSHIFT is pictorially shown in Fig.4

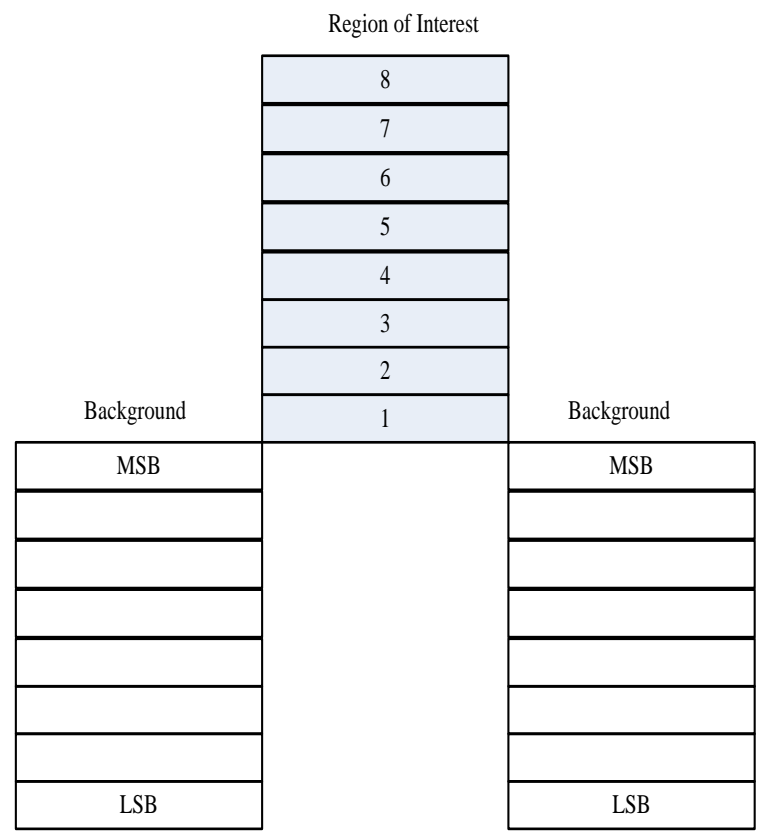

Fig 4: Adopted MAXHIFT Method

The study uses MAXSHIFT method where the scaling value is evaluated to posses the random shape region of interest without any requirement for transmitting shape information to the decoding end. Hence, the study considers that adopting MAXSHIFT method ensures no reason for decoding module to generate a mask for region of interest. The system performs mapping of the selected region of interest from the spatial domain to wavelet transforms domain that is dependent on the deployed wavelet filters, and it is normalized for various polygonal regions. The system performs reading of the quantized coefficients and selects a scaling value $S$ so that the minimal value of the coefficient belonging to the region of interest is maximum than the higher value of the coefficient of the non region of the interest portion of the input medical image. In this technique, the entire wavelet coefficients belonging to a background of the image is scaled down by a constant $\mathrm{D}$ in a degree of $(\mathrm{S}+\mathrm{D})$. Therefore, all the coefficients of the wavelet transform that corresponds to the non-region of the interest portion is considered to have a magnitude less than 1. After the bitstream is received in the decoder end, the system scales up all the wavelet coefficient that have magnitude more than 1 , which ensures that no extra information about the shape of the region of interest is needed.

After performing MAXSHIFT operation, the residual outcome of the study is subjected to entropy encoding that is a lossless data compression scheme that is independent of the specific characteristics of the medium. The study considers usage of either Huffman coding or Arithmetic coding. Huffman coding deploys a particular process for selecting the depiction for each symbol, resulting in a prefix code that represents the most general source symbols using shorter strings of bits than are used for less common source symbols. However, arithmetic coding differs from other forms of entropy encoding such as Huffman coding, which rather than separating the input into component symbols and replacing each with a code, arithmetic coding encodes the entire message into a single number, a fraction $n$ where $(0.0 \leqslant n<1.0)$. Once done, the decoding operation is performed using Inverse DWT and same entropy decoding style to accomplish the outcome of the image for evaluating the SNR and BPP.

\section{IMPLEMENTATION AND RESULT DISCUSSION}

The proposed algorithm is simulated. The proposed implementation has been done considering the database of Cornell University Vision and Image Analysis Group [24]. (http://www.via.cornell.edu/databases/)( http://www.isi.uu.nl/Research/Databases/SCR/download.php)

The algorithm implementation of the proposed study is as follows:

Algorithm: Performing Compression of Medical Image using ROI based approach

Input: Input medical image

Output: SNR, BPP

Steps

1. Input medical Image $I_{M}$ and find its size $I_{\text {size }}$.

2. Estimate the maximum level $=\log _{2}\left(I_{\text {size }}\right)$.

3. Apply polygonal shape region-of-interest technique.

[consider $\mathrm{I}_{\mathrm{ROI}}$ is selected ROI image]

4. Apply 2 dimensional DWT on horizontal, vertical and diagonal and estimate wavelet coefficients.

5. Initialize a new matrix to store the coefficient values.

6. Scale the $\mathrm{I}_{\mathrm{ROI}}$ matrix \& obtain the level coefficient.

7. Apply morphological operation on the $\mathrm{I}_{\mathrm{ROI}}$. 


\section{Perform MAXSHIFT operation on each coefficient}

-create a function MAXSHIFT with input arguments=wavelet coefficients, $\mathrm{I}_{\mathrm{ROI}}$.

-Evaluate the background of the $\mathrm{I}_{\mathrm{bg}}$

-For 8 bit planes, estimate the scaling factor $\left(\mathrm{S}=\max \left(\max \left(\mathrm{I}_{\mathrm{bg}}\right)\right)\right)$.

-Perform shifting bits with respect to 1-8 bits.

9. Arrange coefficient to the image file

10. Perform Entropy Encoding

Switch (option)

\{Option-1: Huffman Encoder

break();

Option-2: Arithmetic Encoder break();

\}

11. Transmit the coefficients and scaling factor.

12. Estimate BPP and SNR

End

The proposed algorithm can be considered as the cost effective as it uses Huffman encoding technique. The running time of Huffman's method is fairly efficient; it takes $\mathrm{O}(\mathrm{n} \log \mathrm{n})$ operations to construct it. The cumulative effectiveness in the results accomplished from the study can be evaluated by testing the total of 20 images and their accomplished SNR and BPP.

Table 2. Result of SNR and BPP

\begin{tabular}{|l|c|c|}
\hline Image Inputs & SNR & BPP \\
\hline Image-1 & 21.1 & 0.44 \\
\hline Image-2 & 37.5 & 0.43 \\
\hline Image-3 & 39.7 & 0.41 \\
\hline Image-3 & 35.9 & 0.32 \\
\hline Image-5 & 29.7 & 0.65 \\
\hline Image-6 & 39.8 & 0.41 \\
\hline Image-7 & 25.6 & 0.56 \\
\hline Image-8 & 35 & 0.47 \\
\hline Image-9 & 59 & 0.41 \\
\hline Image-10 & 42.3 & 0.39 \\
\hline Image-11 & 30.12 & 0.32 \\
\hline Image-12 & 29.2 & 0.47 \\
\hline Image-13 & 27.1 & 0.35 \\
\hline Image-14 & 23.7 & 0.41 \\
\hline Image-15 & 21.4 & 0.41 \\
\hline Image-16 & 25.9 & 0.32 \\
\hline Image-17 & 18.8 & 0.47 \\
\hline Image-18 & 19.3 & 0.47 \\
\hline Image-19 & 23.6 & 0.42 \\
\hline Image-20 & 21.7 & 0.31 \\
\hline Average Value & 30.321 & 0.422 \\
\hline & & \\
\hline & & (1) \\
\hline
\end{tabular}

Usage of 20 sample images was tested on the proposed study and mean SNR as well as bits-per-pixel values were significantly extracted and analyzed. However, the trend and relationship of the proposed model is also interested to identify, how using MAXSHIFT technique, the compression technique can be further enhanced. Hence, the proposed study is also evaluated with respect to the compression ratio. The cumulative outcome of this part of the evaluation is highlighted in Table 3.

Table 3. Trend of bpp for full and ROI image

\begin{tabular}{|l|l|l|l|l|}
\hline & \multicolumn{2}{|c|}{ Full Image } & \multicolumn{2}{c|}{ ROI Image } \\
\hline BPP & PSNR & CR & PSNR & CR \\
\hline 0.0625 & 26.285 & 256.13 & 34.28 & 20.148 \\
\hline 0.1250 & 27.058 & 127.85 & 35.02 & 18.293 \\
\hline 0.2500 & 28.846 & 44.10 & 35.61 & 14.364 \\
\hline 0.3000 & 29.105 & 30.72 & 36.52 & 12.042 \\
\hline 0.4000 & 30.520 & 24.87 & 36.76 & 11.983 \\
\hline 0.5000 & 31.090 & 20.01 & 37.03 & 10.195 \\
\hline 0.7000 & 32.183 & 17.93 & 37.98 & 8.983 \\
\hline 0.8000 & 32.962 & 15.84 & 39.01 & 7.619 \\
\hline 0.9000 & 33.085 & 14.67 & 39.81 & 6.649 \\
\hline 1.0000 & 34.293 & 10.09 & 40.29 & 4.643 \\
\hline
\end{tabular}

Table 3 highlights the trend of variation in association with two factors i) bits-per-pixel values with PSNR of both full image and region of interest and ii) bits-per-pixel values with compression ratio of both full and region of interest. The first trend can be observed in Fig.5.

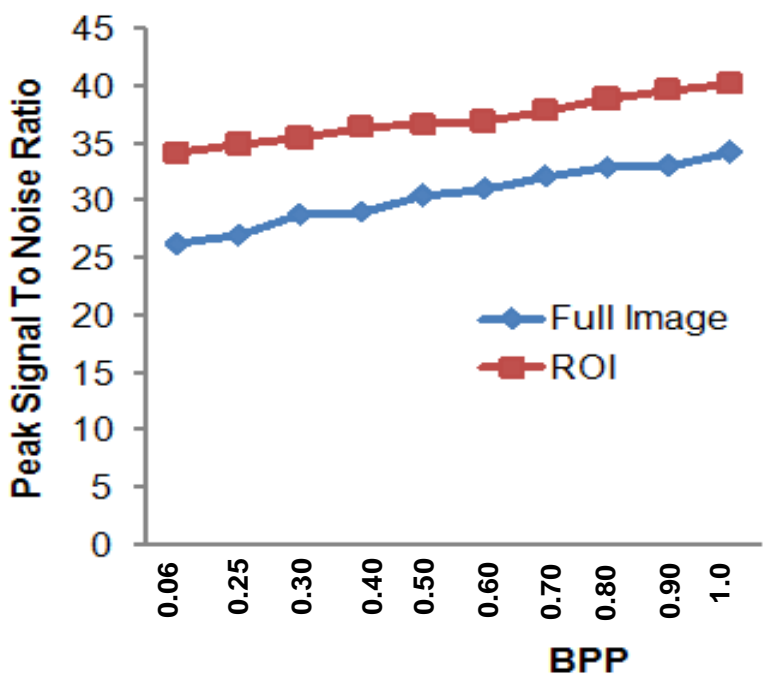

Fig 5: PSNR vs BPP

The outcome exhibited in Fig.5 indicates that peak signal to noise ratio increases with the increase of bits-per-pixel value, which essentially means that amount of the signal increases with increase of image resolution expressed in terms of bits-perpixel. It can be seen that for full medical image, PSNR has stiff increment with the increase of bits-per-pixel. The curve starts from $25 \mathrm{~dB}$ PSNR values and increases to more than $30 \mathrm{db}$ of PSNR with increase of bits-per-pixel values. However, the MAXSHIFT procedure is not applied on the entire image and it is only applied on region of interest, for which reason, once the 
image is cropped from full image by the user, the bit-level of the information for the background drastically decreases. Application of two-dimension discreet wavelength transform results in restoring the co-efficient of region of interest only thereby ensuring better decomposition level of the signals. Hence, peak-signal-to noise ratio for region of interest image is considerably higher compared to that of full image with respect to increase of bits-per-pixel.

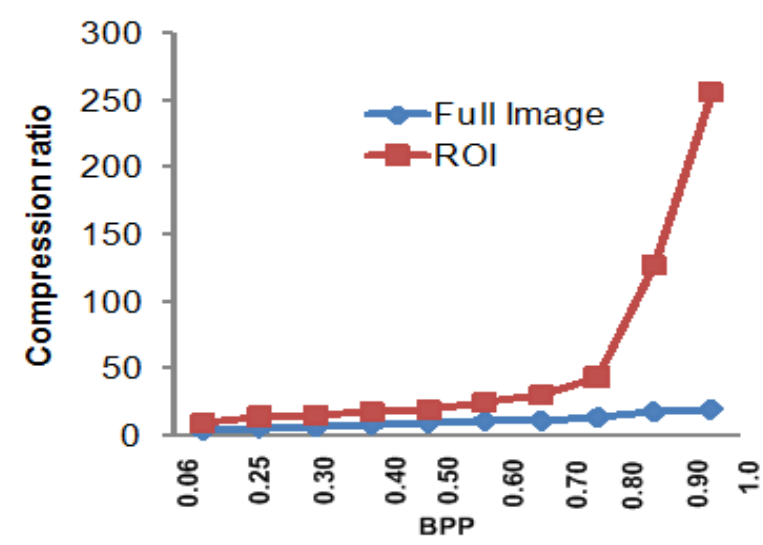

Fig 6: Compression Ratio vs BPP

Fig.6 discusses about the trend of variation in compression ratio with respect to bits-per-pixel. The compression ratio parameter is selected for minimizing the irrelevancy as well as redundancy of the image data for the purpose of storing as well as transmitting the precise data in an efficient form. Usually, the compression ratio techniques that are applied in the proposed system calls for higher amount of processing power for performing encoding as well as decoding. Hence, efficiency evaluation of the proposed compression scheme also requires considering compression ratio parameter. The outcome of the study exhibited in Fig. 6 shows that compression ratio for full image is comparatively minimal with respect to that of region of interest. The proposed study achieves exponentially higher compression ratio using MAXSHIFT as it is adopted a technique using wavelets that are essentially associated with relative insufficiency of the wavelet domain representation for the signal. The proposed algorithm is designed based on the frequency of occurrences of the pixels in image that was eventually deployed for using a minimal number of bits for performing encoding of the data that occurs more frequently. The enhance MAXSHIFT principle can now consider taking the benefit of redundancies such as array of elements ending with a long list of zeros by inserting a new code in the place of frequent combination of numbers.

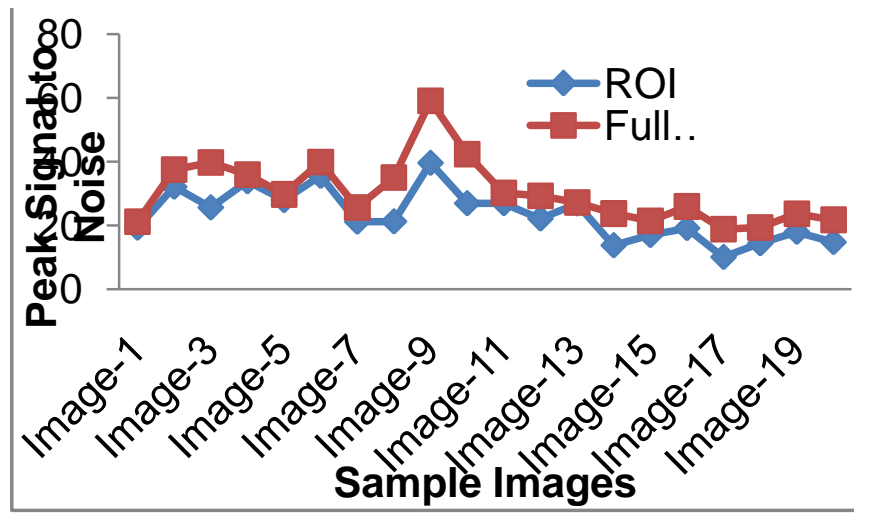

Fig 7: Evaluation of PSNR

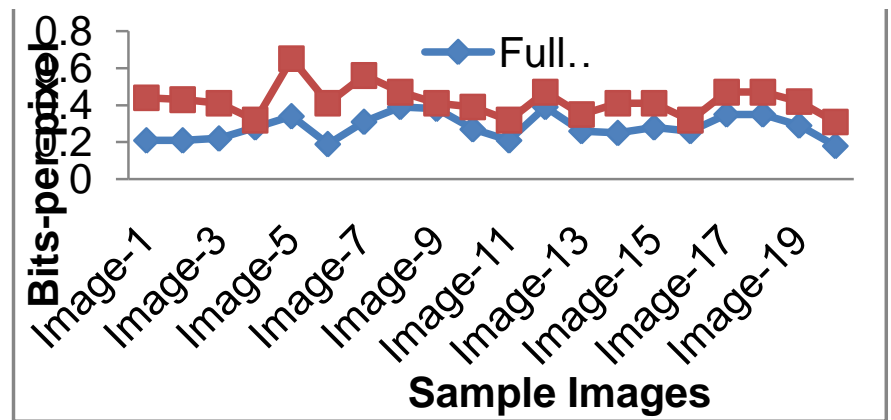

Fig 8: Evaluation of Bits-Per-Pixel

The Table 2 shows the results of signal-to-noise ratio and bitsper-pixel, which will represent the quality of the medical images from the output viewpoint. The values specified in the table could be analyzed individually as shown in Fig.5 and Fig.6. Hence, it can be seen that the average SNR of the 20 different input image is estimated to be 30.32 whereas bits per pixel (BPP) is found to be 0.422 . Hence, it can be said that proposed system has generated a better results. It is already known that typical values of the SNR in the lossy medical images are usually in between 30 to 50 decibel provided that the color (bit) depth is about 8 bit where accomplishment of higher value is supposed to give better result. Hence, it can be only said that the proposed outcome of SNR is in the acceptable range, which could be possible enhanced in future along with more visual quality. Hence, the comparative analysis shows that it is highly feasible to apply enhance MAXSHIFT technique integrated with transform technique to create better compression scheme for medical images. The outcome exhibited in Fig.5 and Fig.6 shows that ROI images posses better signal contents as well as better resolution in terms of bits-per-pixels.

\section{CONCLUSION}

The proposed system has used the MAXSHIFT method to carry out the medical image compression using region of interest technique. One of the advantages of the proposed system is that it permits the encoding of the randomly shaped region of interest without the necessity of estimating masking factor of the region of interest. With the use of Huffman like entropy coding standard, the algorithm has become very much cost effective with less time and space complexity. The design of the encoder is very much uncomplicated as no shape encoding was done (or needed). The design of the decoding scheme is also associated with the generation of SNR to estimate the outcome of the study. Since, compression leads to loss of significant information, so the outcome of the study is evaluated with respect to SNR and BPP to identify that the system generates enough signal and has better perceptual visual quality. The paper has discussed that MAXSHIFT has been seen to be adopted by various researchers in the past, so the proposed system chose to implement the method to check the estimated outcome of the results. However, the benchmarking of the proposed study is required. Hence, the future direction of the present work will be to explore an efficient mathematical technique which can probably act as a substitute of MAXSHIFT method. The aim of the future work will be to benchmark the future outcome with the standard work of MAXSHIFT present in the current paper. Although, the proposed method gives good compression without losing the features but to achieve the computational performance there is need of fast computational process Hence, our future work direction will be to use mathematical approach for achieving better computational capability while accomplishing efficient compression using region of interest. 


\section{REFERENCES}

[1] Radha, V.2011. A Comparative Study on ROI-Based Lossy Compression Techniques for Compressing Medical Image. Proceedings of the World Congress on Engineering and Computer Science, Vol I

[2] Gokturk,S.B., Tomasi. C., Girod, B, Beaulieu, C.2001. Medical image compression based on region of interest with application to colon CT images. Engineering in Medicine and Biology Society. Proceedings of the 23rd Annual International Conference of the IEEE, Vol.3, No. pp.2453-2456

[3] Loganathan, R., Kumaraswamy, Y.S.2012. Medical image compression with lossless region of interest using adaptive active contour. Journal of Computer Science, Vol. 8, pp. 747-751

[4] Babu, D., Vijendra, D.N., Alamelu.2009. Wavelet Based Medical Image Compression Using ROI EZW. International Journal of Recent Trends in Engineering, Vol.1, No.3, pp. 97-100

[5] Bairagi, V.K., Sapkal, A.M.2013. ROI-based DICOM image compression for telemedicine. Vol. 38, pp. 123131

[6] Sridevi, M.E., Vijayakuymar, V.R., Anuja, R.2012. A Survey on Various Compression Methods for Medical Images. International Journal of Intelligent Systems and Applications (IJISA), Vol 4, No.3

[7] Wakatani, A.2002. Digital watermarking for ROI medica images by using compressed signature image. System Sciences, Proceedings of the 35th Annual Hawaii International Conference

[8] Kumar, S., Goel, N., Singh, V., Choudhary, A., Sorohi, N., Singh, G.2013. Fast and Efficient Medical Image Compression Using Contourlet Transform:(FEMI-CCT) Open Journal of Computer Sciences, Vol.1, No. 1

[9] Thomas, D.S., Moorthi, M., Muthalagu, R.2014. Medical Image Compression based on Automated ROI Selection for Telemedicine Application. International Journal Of Engineering And Computer Science. Vol. 3, Issue. 1

[10] Chavad, P., Patel, N., Patel, K.2014. Region of Interest Based Image Compression. International Journal of Innovative Research in Computer and Communication Engineering. Vol.2, Issue. 1

[11] Naidu, V.R., Agrawal, V.K., Ramakrishnan, A.G.2008. MR image compression with ROI decoding, SNR and Spatial scalability, In proceeding of: IISc Centenary Conference

[12] Arun, R., Murugan, D.2012. Medical Image Compression Using Region Growing Segmentation. International Journal Of Computational Engineering Research. Vol.2, Issue. 2
[13] Kaur, B., Aggarwal, D., Kaur, G., Kaur, A.2011. Efficient Image Compression based on Region of Interest, International Journal of Computer Science and Telecommunications, Vol.2, Issue. 1

[14] Sridevi, S., Vijayakumar, V.R.2013. Contextual Region of Interest Based Medical Image Compression using Contextual Listless SPIHT Algorithm for Brain Images. International Journal of Engineering \& Technology, pp. 0975-4024

[15] Lavanya, M., Kumar, S.2013. Intelligent Compression of Medical Images Based on Multi ROI. International Journal of Emerging Technology and Advanced Engineering, Vol.3, Issue.1

[16] Mateika, D., Martaviciu, R.2006. Analysis of the Compression Ratio and Quality in Medical Images. Information Technology and Control, Vol.35, No.4

[17] N-A, Amine., C-M. Christine2013.Compression of Biomedical Images and Signals, John Wiley \& Sons Science

[18] Beutel, J.2000. Handbook of Medical Imaging: Display and PACS, SPIE Press, pp.512

[19] Penedo, M.., Pearlman, W.A., Tahoces, P.G., Souto, M., Vidal, J.J.2003. Region-based wavelet coding methods for digital mammography.IEEE Trans Med Imaging. Vol.22

[20] Silva, V.F.2010. Advances in medical image compression: Novel schemes for highly efficient storage, transmission and on demand scalable access for 3D and 4D medical imaging data. Doctorial Thesis, The University of British Columbia

[21] Anastassopoulos, G.K and Skodras, A.N.2002.JPEG2000 ROI Coding In Medical Imaging Applications. Proc. 2nd LASTED Int. Conf. On Visualisation, Imaging and Image Processing, pp. 783-788

[22] Chen, Y-T., Tseng, D-C and Chang, P-C.2006. WaveletBased Image Compression with Polygon-Shaped Region of Interest, Springer, pp. $878-887$

[23] Pawadshetty, J.S., Bakal, J.W.2013.JPEG 2000 Region of Interest Coding Methods. International Journal of Engineering Research and Applications, Vol. 3, Issue. 1, pp.1184-1188

[24] http://www.via.cornell.edu/

[25] Doukas, C., Maglogiannis, I.2007. Region of Interest Coding Techniques for Medical Image Compression.IEEE Engineering In Medicine And Biology Magazine

[26] Suma and V. Sridhar.2014.Article: A Review of the Effective Techniques of Compression in Medical Image Processing. International Journal of Computer Applications, Vol. 97(6), pp.23-30 